\title{
A method for fractional Volterra integro-differential equations by Laguerre polynomials
}

\author{
Dilek Varol Bayram ${ }^{1}$ and Ayşegül Daşcıoğlu ${ }^{1 *}$ (D)
}

"Correspondence:

aakyuz@pau.edu.tr

${ }^{1}$ Department of Mathematics,

Faculty of Science and Arts,

Pamukkale University, Denizli,

Turkey

\begin{abstract}
The main purpose of this study is to present an approximation method based on the Laguerre polynomials for fractional linear Volterra integro-differential equations. This method transforms the integro-differential equation to a system of linear algebraic equations by using the collocation points. In addition, the matrix relation for Caputo fractional derivatives of Laguerre polynomials is also obtained. Besides, some examples are presented to illustrate the accuracy of the method and the results are discussed.
\end{abstract}

Keywords: Volterra integro-differential equations; Laguerre polynomials; Fractional integro-differential equations

\section{Introduction}

The fractional calculus represents a powerful tool in applied mathematics to study numerous problems from different fields of science and engineering such as mathematical physics, finance, hydrology, biophysics, thermodynamics, control theory, statistical mechanics, astrophysics, cosmology, and bioengineering [1]. Since the fractional calculus has attracted much more interest among mathematicians and other scientists, the solutions of the fractional differential and integro-differential equations have been studied frequently in recent years [2-10]. The methods that are used to find the solutions of the fractional Volterra integro-differential equations are given as Adomian decomposition [11], Bessel collocation [12, 13], CAS wavelets [14], Chebyshev pseudo-spectral [15], cubic B-spline wavelets [16], Euler wavelet [17], fractional differential transform [18], homotopy analysis [19], homotopy perturbation [20-23], Jacobi spectral-collocation [24, 25], Legendre collocation [26], Legendre wavelet [27], linear and quadratic interpolating polynomials [28], modification of hat functions [29], multi-domain pseudospectral [30], normalized systems functions [31], novel Legendre wavelet Petrov-Galerkin method [32], operational Tau [33], piecewise polynomial collocation [34], quadrature rules [35], reproducing kernel [36], second Chebyshev wavelet [37], second kind Chebyshev polynomials [38], sinccollocation [39, 40], spline collocation [41], Taylor expansion [27], and variational iteration $[20,23]$.

Laguerre polynomials are used to solve some integer order integro-differential equations. These equations are given as Altarelli-Parisi equation [42], Dokshitzer-Gribov-

(c) The Author(s) 2018. This article is distributed under the terms of the Creative Commons Attribution 4.0 International License (http://creativecommons.org/licenses/by/4.0/), which permits unrestricted use, distribution, and reproduction in any medium, provided you give appropriate credit to the original author(s) and the source, provide a link to the Creative Commons license, and indicate if changes were made. 
Lipatov-Altarelli-Parisi equation [43], pantograph-type Volterra integro-differential equation [44], linear Fredholm integro-differential equation [45, 46], linear integrodifferential equation [47], parabolic-type Volterra partial integro-differential equation [48], nonlinear partial integro-differential equation [49], delay partial functional differential equation [50]. Besides, Laguerre polynomials are used to solve the fractional Fredholm integro-differential equation [51]. However, there has not been a method in the literature for fractional Volterra integro-differential equations in terms of Laguerre polynomials. That is why, in this paper, a method based on the Laguerre polynomials is presented to find the solutions of linear fractional Volterra integro-differential equation in the form

$$
D^{\alpha} y(x)+p(x) y(x)=g(x)+\lambda \int_{0}^{x} K(x, t) y(t) d t, \quad 0 \leq x \leq b, \alpha>0
$$

with the initial conditions

$$
y^{(j)}(0)=c_{j}, \quad j=0,1, \ldots, n-1, \text { and } n-1<\alpha<n .
$$

Here, $n \in \mathbb{Z}^{+}, \lambda \in \mathbb{R}, K(x, t), p(x)$, and $g(x)$ are given functions, $y(x)$ is the unknown function to be determined, $D^{\alpha} y(x)$ indicates the Caputo fractional derivative of $y(x)$. Now, we give the definition and the basic properties of the Caputo fractional derivative as follows.

Definition ([52]) The Caputo fractional differentiation operator $D^{\alpha}$ of order $\alpha$ is defined as follows:

$$
D^{\alpha} f(x)=\frac{1}{\Gamma(n-\alpha)} \int_{0}^{x} \frac{f^{(n)}(t)}{(x-t)^{\alpha+1-n}} d t, \quad \alpha>0,
$$

where $n-1<\alpha<n, n \in \mathbb{Z}^{+}$.

Besides, the Caputo fractional derivative of a constant function is zero and the Caputo fractional differentiation operator is linear [53].

The aim of this study is to give an approximate solution of problem (1)-(2) in the form

$$
y(x) \cong y_{N}(x)=\sum_{i=0}^{N} a_{i} L_{i}(x),
$$

where $a_{i}$ are unknown coefficients, $N$ is chosen any positive integer such that $N \geq n$, and $L_{i}(x)$ are the Laguerre polynomials of order $i$ defined in Ref. [54] as

$$
L_{i}(x)=\sum_{k=0}^{i}(-1)^{k} \frac{i !}{(i-k) !(k !)^{2}} x^{k} .
$$

Besides, the main purpose of the solution method presented in this paper is to obtain the Caputo fractional derivative of the Laguerre polynomials in terms of the Laguerre polynomials and to give a matrix representation for this relation. The Caputo fractional derivative of the Laguerre polynomials is mentioned in Ref. [51,55-57]. While these matrix relations have been given depending on approximate matrices, the relation proposed in this paper is new, exact, and simpler than the former ones. 
This paper is organized as follows: In Sect. 2, the main matrix relations of the terms in Eq. (1) are established. In Sect. 3, the collocation method which is used to find the solution is introduced. In Sect. 4, some numerical examples are solved and their comparison with the existing results in the literature are presented to verify the accuracy and efficiency of the proposed method. The conclusion is given in Sect. 5 .

\section{Main matrix relations}

In this section, we construct the matrix forms of each term of Eq. (1). Firstly, we can write the approximate solution (3) in the matrix form

$$
y_{N}(x)=\mathbf{L}(x) \mathbf{A},
$$

where

$$
\mathbf{L}(x)=\left[\begin{array}{llll}
L_{0}(x) & L_{1}(x) & \cdots & L_{N}(x)
\end{array}\right] \text { and } \mathbf{A}=\left[\begin{array}{llll}
a_{0} & a_{1} & \cdots & a_{N}
\end{array}\right]^{T} .
$$

Now, we will state a theorem that gives the Caputo fractional derivative of Laguerre polynomials in terms of Laguerre polynomials.

Theorem Let $L_{i}(x)$ be Laguerre polynomial of order $i$, then the Caputo fractional derivative of $L_{i}(x)$ in terms of Laguerre polynomials is found as follows:

$$
D^{\alpha} L_{i}(x)=0, \quad i<\lceil\alpha\rceil,
$$

and otherwise

$$
D^{\alpha} L_{i}(x)=x^{1-\alpha} \sum_{k=\lceil\alpha\rceil}^{i} \sum_{j=0}^{k-1}(-1)^{j+k} \frac{(k-1) !}{\Gamma(k+1-\alpha)}\left(\begin{array}{c}
i \\
k
\end{array}\right)\left(\begin{array}{c}
k-1 \\
j
\end{array}\right) L_{j}(x),
$$

where $\lceil\alpha\rceil$ denotes the ceiling function which is the smallest integer greater than or equal to $\alpha$.

Proof Let us begin deriving the Laguerre polynomials with the definition of them:

$$
D^{\alpha} L_{i}(x)=D^{\alpha}\left\{\sum_{k=0}^{i}(-1)^{k} \frac{i !}{(i-k) !(k !)^{2}} x^{k}\right\} .
$$

By the linearity of Caputo fractional derivative, we get

$$
D^{\alpha} L_{i}(x)=\sum_{k=0}^{i}(-1)^{k} \frac{i !}{(i-k) !(k !)^{2}} D^{\alpha}\left(x^{k}\right) .
$$

Using the Caputo fractional derivative of $x^{k}, k=0,1,2, \ldots$,

$$
D^{\alpha} x^{k}= \begin{cases}0, & k<\lceil\alpha\rceil, \\ \frac{\Gamma(k+1)}{\Gamma(k+1-\alpha)} x^{k-\alpha}, & k \geq\lceil\alpha\rceil,\end{cases}
$$


we obtain $D^{\alpha} L_{i}(x)=0$ for $i<\lceil\alpha\rceil$ and

$$
D^{\alpha} L_{i}(x)=\sum_{k=\lceil\alpha\rceil}^{i} \frac{(-1)^{k}}{\Gamma(k+1-\alpha)}\left(\begin{array}{l}
i \\
k
\end{array}\right) x^{k-\alpha}, \quad i=\lceil\alpha\rceil,\lceil\alpha\rceil+1 .
$$

At this step, by taking $x^{1-\alpha}$ out of the series and using the Laguerre series of the function $x^{k}$ given by Lebedev [58]

$$
x^{k}=k ! \sum_{j=0}^{k}(-1)^{j}\left(\begin{array}{l}
k \\
j
\end{array}\right) L_{j}(x), \quad 0<x<\infty, k=0,1,2 \ldots
$$

we have relation (5) and the proof is completed.

\subsection{Matrix relation for the differential part}

Now, we will write the matrix form of the differential part of Eq. (1). The fractional part is obviously seen as

$$
D^{\alpha} \mathbf{L}(x)=\left[\begin{array}{llll}
D^{\alpha} L_{0}(x) & D^{\alpha} L_{1}(x) & \cdots & D^{\alpha} L_{N}(x)
\end{array}\right] .
$$

The right-hand side of this equation can be expressed as

$$
D^{\alpha} \mathbf{L}(x)=x^{1-\alpha} \mathbf{L}(x) \mathbf{S}_{\alpha}
$$

where $\mathbf{S}_{\alpha}$ is an $(N+1)$ dimensional square matrix denoted by

$$
\mathbf{S}_{\alpha}=\left[\begin{array}{ccccc}
0 & S_{1,1} & \left(\begin{array}{c}
1 \\
0
\end{array}\right) S_{1,2}+\left(\begin{array}{c}
2 \\
0
\end{array}\right) S_{2,2} & \cdots & \sum_{k=1}^{N}\left(\begin{array}{c}
k-1 \\
0
\end{array}\right) S_{k, N} \\
0 & 0 & -\left(\begin{array}{c}
1 \\
1
\end{array}\right) S_{2,2} & \cdots & -\sum_{k=2}^{N}\left(\begin{array}{c}
k-1 \\
1
\end{array}\right) S_{k, N} \\
0 & 0 & 0 & \cdots & \sum_{k=3}^{N}\left(\begin{array}{c}
k-1 \\
2
\end{array}\right) S_{k, N} \\
\vdots & \vdots & \vdots & \ddots & \vdots \\
0 & 0 & 0 & \cdots & (-1)^{N} S_{N, N} \\
0 & 0 & 0 & \cdots & 0
\end{array}\right] .
$$

Here, the $S_{k, i}$ terms in the entries of $\mathbf{S}_{\alpha}$ are defined as follows:

$$
S_{k, i}= \begin{cases}(-1)^{k} \frac{(k-1) !}{\Gamma(k+1-\alpha)}\left(\begin{array}{l}
i \\
k
\end{array}\right), & \text { if }\lceil\alpha\rceil \leq k \leq i, \\
0, & \text { otherwise. }\end{cases}
$$

Then, by using relations (4) and (7), the fractional differential part of Eq. (1) can be expressed as

$$
D^{\alpha} y(x) \cong D^{\alpha} \mathbf{L}(x) \mathbf{A}=x^{1-\alpha} \mathbf{L}(x) \mathbf{S}_{\alpha} \mathbf{A} .
$$

\subsection{Matrix relation for conditions}

The relation between $\mathbf{L}(x)$ and its derivatives of integer order is given by Yüzbaşı[44] as

$$
\mathbf{L}^{(i)}(x)=\mathbf{L}(x) \mathbf{M}^{i}, \quad i=0,1,2, \ldots
$$


where the matrix $\mathbf{M}$ is defined by

$$
\mathbf{M}=\left[\begin{array}{ccccc}
0 & -1 & -1 & \cdots & -1 \\
0 & 0 & -1 & \cdots & -1 \\
0 & 0 & 0 & \cdots & -1 \\
\vdots & \vdots & \vdots & \ddots & \vdots \\
0 & 0 & 0 & \cdots & -1 \\
0 & 0 & 0 & \cdots & 0
\end{array}\right]
$$

By using relation (9), the corresponding matrix forms of the conditions defined in (2) can be written as

$$
y^{j}(0) \cong \mathbf{L}(0) \mathbf{M}^{j} \mathbf{A}=c_{j}, \quad j=0,1, \ldots, n-1 .
$$

Here, the matrix $\mathbf{L}(0) \mathbf{M}^{j}$ is named $\mathbf{U}_{j}$ where it is an $1 \times(N+1)$ dimensional matrix. Hence, Eq. (10) becomes

$$
\mathbf{U}_{\mathrm{j}} \mathbf{A}=c_{j}, \quad j=0,1, \ldots, n-1 .
$$

\section{Method of solution}

To obtain the approximate solution of Eq. (1), we compute the unknown coefficients by using the following collocation method. Firstly, let us substitute the matrix forms (4) and (8) into Eq. (1), and thus we obtain the matrix equation

$$
x^{1-\alpha} \mathbf{L}(x) \mathbf{S}_{\alpha} \mathbf{A}+p(x) \mathbf{L}(x) \mathbf{A}=g(x)+\lambda \int_{0}^{x} K(x, t) \mathbf{L}(t) \mathbf{A} d t
$$

By substituting the collocation points $x_{s}>0(s=0,1, \ldots, N)$ into Eq. (11), we have a system of matrix equations

$$
\left\{x_{s}^{1-\alpha} \mathbf{L}\left(x_{s}\right) \mathbf{S}_{\alpha}+p\left(x_{s}\right) \mathbf{L}\left(x_{s}\right)-\lambda \mathbf{v}\left(x_{s}\right)\right\} \mathbf{A}=g\left(x_{s}\right)
$$

where $\mathbf{v}\left(x_{s}\right)=\int_{0}^{x_{s}} K\left(x_{s}, t\right) \mathbf{L}(t) d t$. This system can be written in the compact form:

$$
\left\{\mathbf{X}_{\alpha} \mathbf{L} \mathbf{S}_{\alpha}+\mathbf{P L}-\lambda \mathbf{V}\right\} \mathbf{A}=\mathbf{G},
$$

where

$$
\begin{array}{cc}
\mathbf{X}_{\alpha}=\left[\begin{array}{cccc}
x_{0}^{1-\alpha} & 0 & \cdots & 0 \\
0 & x_{1}^{1-\alpha} & \cdots & 0 \\
\vdots & \vdots & \ddots & \vdots \\
0 & 0 & \cdots & x_{N}^{1-\alpha}
\end{array}\right], \quad \mathbf{P}=\left[\begin{array}{cccc}
p\left(x_{0}\right) & 0 & \cdots & 0 \\
0 & p\left(x_{1}\right) & \cdots & 0 \\
\vdots & \vdots & \ddots & \vdots \\
0 & 0 & \cdots & p\left(x_{N}\right)
\end{array}\right], \\
\mathbf{L}=\left[\begin{array}{c}
\mathbf{L}\left(x_{0}\right) \\
\mathbf{L}\left(x_{1}\right) \\
\vdots \\
\mathbf{L}\left(x_{N}\right)
\end{array}\right], \quad \mathbf{V}=\left[\begin{array}{c}
\mathbf{v}\left(x_{0}\right) \\
\mathbf{v}\left(x_{1}\right) \\
\vdots \\
\mathbf{v}\left(x_{N}\right)
\end{array}\right], \quad \mathbf{G}=\left[\begin{array}{c}
g\left(x_{0}\right) \\
g\left(x_{1}\right) \\
\vdots \\
g\left(x_{N}\right)
\end{array}\right] .
\end{array}
$$


Denoting the expression in parenthesis of Eq. (13) by $\mathbf{W}$, the fundamental matrix equation for Eq. (1) is reduced to $\mathbf{W A}=\mathbf{G}$, which corresponds to a system of $(N+1)$ linear algebraic equations with unknown Laguerre coefficients $a_{0}, a_{1}, \ldots, a_{N}$.

Finally, to obtain the solution of Eq. (1) under conditions (2), we replace or stack the $n$ rows of the augmented matrix $[\mathbf{W} ; \mathbf{G}]$ with the rows of the augmented matrix $\left[\mathbf{U}_{\mathrm{j}} ; c_{j}\right]$. In this way, the Laguerre coefficients are determined by solving the new linear algebraic system.

\section{Numerical examples}

In this section, we apply the proposed method to four examples existing in the literature and to a test example constructed for this method. We have performed all of the numerical computations using Mathcad 15. We also use the collocation points by using the formula $x_{s}=\left[1-\cos \left(\frac{(s+1) \pi}{N+1}\right)\right] / 2, s=0,1, \ldots, N$.

Example 1 Consider the following fractional integro-differential equation:

$$
D^{\frac{1}{2}} y(x)=y(x)+\frac{8}{3 \Gamma(0.5)} x^{1.5}-x^{2}-\frac{1}{3} x^{3}+\int_{0}^{x} y(t) d t
$$

subject to $y(0)=0$ with the exact solution $y(x)=x^{2}$.

Applying the procedure in Sect. 3, the main matrix equation of this problem and the conditions are given by

$$
\left\{\mathbf{X}_{1 / 2} \mathbf{L} \mathbf{S}_{1 / 2}-\mathbf{L}-\mathbf{V}\right\} \mathbf{A}=\mathbf{G}
$$

and

$$
\mathbf{U}_{0} \mathbf{A}=0 .
$$

If we take $N=2$, the collocation points become $x_{0}=0.25, x_{1}=0.75, x_{2}=1$. Then the matrices mentioned above are

$$
\begin{array}{ll}
\mathbf{X}_{1 / 2}=\left[\begin{array}{ccc}
\frac{1}{2} & 0 & 0 \\
0 & \frac{\sqrt{3}}{2} & 0 \\
0 & 0 & 1
\end{array}\right], & \mathbf{L}=\left[\begin{array}{ccc}
1 & \frac{3}{4} & \frac{17}{32} \\
1 & \frac{1}{4} & -\frac{7}{32} \\
1 & 0 & -\frac{1}{2}
\end{array}\right], \\
\mathbf{V}=\left[\begin{array}{ccc}
\frac{1}{4} & \frac{7}{32} & \frac{73}{384} \\
\frac{3}{4} & \frac{15}{32} & \frac{33}{128} \\
1 & \frac{1}{2} & \frac{1}{6}
\end{array}\right], & \mathbf{G}=\left[\begin{array}{c}
\frac{1}{3 \sqrt{\pi}}-\frac{13}{192} \\
\frac{\sqrt{3}}{\sqrt{\pi}}-\frac{45}{64} \\
\frac{8}{3 \sqrt{\pi}}-\frac{4}{3}
\end{array}\right],
\end{array}
$$

By solving this system, we get $a_{0}=2, a_{1}=-4, a_{2}=2$. When we substitute the determined coefficients into Eq. (3), we get the exact solution.

Using the homotopy analysis method, this problem was also solved by Awawdeh et al. [19]. They found the approximate solution for $N=5$, but they did not state the numerical results of the errors of their method. Besides, Sahu et al. [32] found the approximate solution with the maximum absolute error $4.2 \times 10^{-15}$ by the Legendre wavelet PetrovGalerkin method for $N=6$. If the results are compared, it can be said that the proposed method is better than the other methods since the exact solution is found for $N=2$. 
Example 2 Consider the following fractional integro-differential equation:

$$
D^{0.75} y(x)=\frac{1}{\Gamma(1.25)} x^{0.25}+(x \cos x-\sin x) y(x)+\int_{0}^{x} x \sin t y(t) d t, \quad 0 \leq x \leq 1,
$$

subject to $y(0)=0$ with the exact solution $y(x)=x$.

Applying the procedure in Sect. 3, the main matrix equation of this problem and the conditions are given by

$$
\left\{\mathbf{X}_{3 / 4} \mathbf{L} \mathbf{S}_{3 / 4}-\mathbf{P L}-\mathbf{V}\right\} \mathbf{A}=\mathbf{G}
$$

and

$$
\mathbf{U}_{0} \mathbf{A}=0 .
$$

If we take $N=1$, the collocation points become $x_{0}=0.5, x_{1}=1$. Then the matrices mentioned above are

$$
\begin{aligned}
& \mathbf{X}_{3 / 4}=\left[\begin{array}{cc}
\frac{\sqrt[4]{8}}{2} & 0 \\
0 & 1
\end{array}\right], \quad \mathbf{L}=\left[\begin{array}{ll}
1 & \frac{1}{2} \\
1 & 0
\end{array}\right], \quad \mathbf{S}_{3 / 4}=\left[\begin{array}{cc}
0 & \frac{2 \sqrt{2} \Gamma\left(\frac{3}{4}\right)}{-\pi} \\
0 & 0
\end{array}\right], \\
& \mathbf{G}=\frac{\Gamma\left(\frac{3}{4}\right)}{\pi}\left[\begin{array}{c}
2^{\frac{5}{4}} \\
2 \sqrt{2}
\end{array}\right], \quad \mathbf{U}_{0}=\left[\begin{array}{ll}
1 & 1
\end{array}\right], \\
& \mathbf{V}=\left[\begin{array}{ccc}
{\left[\sin \left(\frac{1}{4}\right)\right]^{2}} & \frac{\sin ^{2}\left(\frac{1}{4}\right)}{2}-\frac{\sin \left(\frac{1}{2}\right)}{2}+\frac{1}{4} \\
1-\cos (1) & \frac{1}{2}-\frac{\sin (1)}{2}
\end{array}\right], \quad \mathbf{P}=\left[\begin{array}{cc}
\frac{\cos \left(\frac{1}{2}\right)}{2}-\sin \left(\frac{1}{2}\right) & 0 \\
0 & \cos (1)-\sin (1)
\end{array}\right] .
\end{aligned}
$$

By solving this system, we get $a_{0}=1, a_{1}=-1$. When we substitute the determined coefficients into Eq. (3), we get the exact solution.

This problem was also solved by Awawdeh et al. [19] with the homotopy analysis method. They found the approximate solution for $N=5$, but they did not state the numerical results of the errors of their method. Besides, Sahu et al. [32] found the approximate solution with the maximum absolute error $1.1 \times 10^{-16}$ by the Legendre wavelet PetrovGalerkin method for $N=6$. If the results are compared, it can be said that the proposed method is better than the other methods since the exact solution is found for $N=1$.

Example 3 Consider the following fractional integro-differential equation:

$$
D^{\sqrt{3}} y(x)=\frac{2}{\Gamma(3-\sqrt{3})} x^{2-\sqrt{3}}+2 \sin x-2 x+\int_{0}^{x} \cos (x-t) y(t) d t,
$$

subject to $y(0)=0, y^{\prime}(0)=0$ with the exact solution $y(x)=x^{2}$.

Applying the solution method given in Sect. 3 , the main matrix equation of this problem and the conditions are given by

$$
\left\{\mathbf{X}_{\sqrt{3}} \mathbf{L} \mathbf{S}_{\sqrt{3}}-\mathbf{V}\right\} \mathbf{A}=\mathbf{G}
$$

and

$$
\mathbf{U}_{0} \mathbf{A}=0 .
$$


Let $N=2$, the collocation points become $x_{0}=0.25, x_{1}=0.75, x_{2}=1$. Here, the matrices in the main matrix relation of this problem are given as follows:

$$
\begin{aligned}
& \mathbf{X}_{\sqrt{3}}=\left[\begin{array}{ccc}
4^{\sqrt{3}-1} & 0 & 0 \\
0 & \left(\frac{4}{3}\right)^{\sqrt{3}-1} & 0 \\
0 & 0 & 1
\end{array}\right], \quad \mathbf{S}_{\sqrt{3}}=\left[\begin{array}{ccc}
0 & 0 & \frac{1}{\Gamma(3-\sqrt{3})} \\
0 & 0 & \frac{-1}{\Gamma(3-\sqrt{3})} \\
0 & 0 & 0
\end{array}\right], \\
& \mathbf{G}=\left[\begin{array}{c}
2 \sin \left(\frac{1}{4}\right)+\frac{2(4) \sqrt{3}-2}{\Gamma(3-\sqrt{3})}-\frac{1}{2} \\
2 \sin \left(\frac{3}{4}\right)+\frac{2\left(\frac{3}{4}\right)^{2}-\sqrt{3}}{\Gamma(3-\sqrt{3})}-\frac{3}{2} \\
2 \sin (1)+\frac{2}{\Gamma(3-\sqrt{3})}-2
\end{array}\right], \quad \mathbf{L}=\left[\begin{array}{ccc}
1 & \frac{3}{4} & \frac{17}{32} \\
1 & \frac{1}{4} & -\frac{7}{32} \\
1 & 0 & -\frac{1}{2}
\end{array}\right], \quad \mathbf{U}_{0}=\left[\begin{array}{lll}
1 & 1 & 1
\end{array}\right], \\
& \mathbf{V}=\left[\begin{array}{lll}
\sin \left(\frac{1}{4}\right) & \cos \left(\frac{1}{4}\right)+\sin \left(\frac{1}{4}\right)-1 & 2 \cos \left(\frac{1}{4}\right)-\frac{7}{4} \\
\sin \left(\frac{3}{4}\right) & \cos \left(\frac{3}{4}\right)+\sin \left(\frac{3}{4}\right)-1 & 2 \cos \left(\frac{3}{4}\right)-\frac{5}{4} \\
\sin (1) & \cos (1)+\sin (1)-1 & 2 \cos (1)-1
\end{array}\right] .
\end{aligned}
$$

By solving this system, we get $a_{0}=2, a_{1}=-4, a_{2}=2$. When we substitute the determined coefficients into Eq. (3), we get the exact solution.

This problem was also solved by Awawdeh et al. [19] and they found the approximate solution by the homotopy analysis method for $N=5$. By the proposed method, we have found the exact solution of the problem for $N=2$. Apparently, our method is better than the other method.

Example 4 Consider the following fractional Volterra integro-differential equation with the given initial condition $y(0)=0$ and with the non-polynomial exact solution $y(x)=x^{3 / 2}$ :

$$
D^{\frac{1}{3}} y(x)=\frac{3 \sqrt{\pi}}{4 \Gamma(13 / 6)} x^{7 / 6}-\frac{2}{63} x^{9 / 2}\left(9+7 x^{2}\right)+\int_{0}^{x}\left(x t+x^{2} t^{2}\right) y(t) d t .
$$

The main matrix equation of this problem and the conditions are given as

$$
\left\{\mathbf{X}_{1 / 3} \mathbf{L} \mathbf{S}_{1 / 3}-\mathbf{V}\right\} \mathbf{A}=\mathbf{G}
$$

and

$$
\mathbf{U}_{0} \mathbf{A}=0 .
$$

The absolute errors of our method are compared with three methods: linear scheme, quadratic scheme, and linear-quadratic scheme for the fractional integro-differential equations of Kumar et al. [28] for $N=5$ in Table 1. It is seen that our method gives better results than the other methods.

Example 5 Consider the following linear fractional Volterra integro-differential equation which is a test problem to the proposed method with a non-polynomial exact solution and with a non-separable kernel:

$$
D^{\frac{1}{2}} y(x)+y(x)=\frac{2}{5}+\frac{3 \sqrt{\pi} x}{4}+x^{\frac{3}{2}}-2 \frac{e^{x^{\frac{7}{2}}}}{5}+\int_{0}^{x} x e^{x t^{2} \sqrt{t}} y(t) d t
$$


Table 1 Comparison of the absolute errors of Example 4 for different methods

\begin{tabular}{lllll}
\hline$x$ & Linear scheme & Quadratic scheme & Linear-quadratic scheme & Our method \\
\hline 0.2 & $9.8 \times 10^{-3}$ & $9.8 \times 10^{-3}$ & $9.8 \times 10^{-3}$ & $2.9 \times 10^{-4}$ \\
0.4 & $1.1 \times 10^{-2}$ & $4.8 \times 10^{-3}$ & $4.9 \times 10^{-3}$ & $7.3 \times 10^{-4}$ \\
0.6 & $1.2 \times 10^{-2}$ & $2.9 \times 10^{-3}$ & $3.2 \times 10^{-3}$ & $9.5 \times 10^{-4}$ \\
0.8 & $1.4 \times 10^{-2}$ & $2.6 \times 10^{-3}$ & $3.5 \times 10^{-3}$ & $8.3 \times 10^{-4}$ \\
1 & $1.9 \times 10^{-2}$ & $3.3 \times 10^{-3}$ & $5.5 \times 10^{-3}$ & $4.5 \times 10^{-4}$ \\
\hline
\end{tabular}

Table 2 Maximum errors of Example 5 for different $N$ values

\begin{tabular}{llllll}
\hline$N$ & 2 & 4 & 6 & 8 & 10 \\
\hline Maximum errors & $1.3 \times 10^{-2}$ & $1.7 \times 10^{-3}$ & $5.5 \times 10^{-4}$ & $2.4 \times 10^{-4}$ & $1.3 \times 10^{-4}$ \\
\hline
\end{tabular}

subject to the initial condition $y(0)=0$ with the exact solution $y(x)=x^{3 / 2}$.

Since the solution is not a polynomial, the exact solution cannot be obtained by the proposed method. That is why approximate solutions are gained and maximum absolute errors of this problem are given in Table 2 for the different $N$ values.

\section{Conclusion}

In this study, a collocation method based on Laguerre polynomials has been developed for solving the fractional linear Volterra integro-differential equations. For this purpose, the matrix relation for the Caputo fractional derivative of the Laguerre polynomials has been obtained for the first time in the literature. Using these relations and suitable collocation points, the integro-differential equation has been transformed into a system of algebraic equations. The method is faster and simpler than the other methods in the literature, and better than the homotopy analysis and Legendre wavelet method.

\section{Acknowledgements}

The authors would like to thank the reviewers for their constructive comments to improve the quality of this work.

\section{Funding}

This work is supported by the Scientific Research Project Coordination Unit of Pamukkale University with numbers 2018KRM002-227 and 2018KRM002-457.

\section{Competing interests}

The authors declare that they have no competing interests.

Authors' contributions

All authors read and approved the final manuscript.

\section{Publisher's Note}

Springer Nature remains neutral with regard to jurisdictional claims in published maps and institutional affiliations.

Received: 13 August 2018 Accepted: 6 December 2018 Published online: 18 December 2018

\section{References}

1. Abbas, S., Benchohra, M., N'Guerekata, G.M.: Advanced Fractional Differential and Integral Equations. Nova Science Publishers, New York (2015)

2. Hajipour, M., Jajarmi, A., Baleanu, D.: An efficient nonstandard finite difference scheme for a class of fractional chaotic systems. J. Comput. Nonlinear Dyn. 13(2), 021013 (2018)

3. Jajarmi, A., Hajipour, M., Mohammadzadeh, E., Baleanu, D.: A new approach for the nonlinear fractional optimal control problems with external persistent disturbances. J. Franklin Inst. 355(9), 3938-3967 (2018)

4. Jajarmi, A., Baleanu, D.: A new fractional analysis on the interaction of HIV with CD4 + T-cells. Chaos Solitons Fractals $113,221-229(2018)$

5. Hesameddini, E., Shahbazi, M.: Hybrid Bernstein block-pulse functions for solving system of fractional integro-differential equations. Int. J. Comput. Math. 95(11), 2287-2307 (2018)

6. Bhrawy, A.H., Zaky, M.A.: An improved collocation method for multi-dimensional space-time variable-order fractional Schrödinger equations. Appl. Numer. Math. 111, 197-218 (2018) 
7. Zaky, M.A.: An improved tau method for the multi-dimensional fractional Rayleigh-Stokes problem for a heated generalized second grade fluid. Comput. Math. Appl. 75, 2243-2258 (2018)

8. Zaky, M.A., Doha, E.H., Taha, T.M., Baleanu, D.: New recursive approximations for variable-order fractional operators with applications. Math. Model. Anal. 23(2), 227-239 (2018)

9. Bhrawy, A.H., Zaky, M.A., Van Gorder, R.A.: A space-time Legendre spectral tau method for the two-sided space-time Caputo fractional diffusion-wave equation. Numer. Algorithms 71(1), 151-180 (2016)

10. Jajarmi, A., Baleanu, D.: Suboptimal control of fractional-order dynamic systems with delay argument. J. Vib. Control 24(12), 2430-2446 (2018)

11. Mittal, R.C., Nigam, R.: Solution of fractional integro-differential equations by Adomian decomposition method. Int. J. Adv. Appl. Math. Mech. 4(2), 87-94 (2008)

12. Yüzbaşı, Ş.: A numerical approximation for Volterra's population growth model with fractional order. Appl. Math Model. 37, 3216-3227 (2013)

13. Parand, K., Nikarya, M.: Application of Bessel functions for solving differential and integro-differential equations of the fractional order. Appl. Math. Model. 38, 4137-4147 (2014)

14. Saaedi, H., Mohseni Moghadam, M.: Numerical solution of nonlinear Volterra integro-differential equations of arbitrary order by CAS wavelets. Commun. Nonlinear Sci. Numer. Simul. 16, 1216-1226 (2011)

15. Sweilam, N.H., Khader, M.M.: A Chebyshev pseudo-spectral method for solving fractional-order integro-differential equations. ANZIAM J. 51, 464-475 (2010)

16. Maleknejad, K., Sahlan, M.N., Ostadi, A.: Numerical solution of fractional integro-differential equation by using cubic B-spline wavelets. In: Proceedings of the World Congress on Engineering 2013, Vol. I, London, UK, 3-5 July 2013 (2013)

17. Wang, Y., Zhu, L.: Solving nonlinear Volterra integro-differential equations of fractional order by using Euler wavelet method. Adv. Differ. Equ. 201727 (2017)

18. Arikoglu, A., Ozkol, l.: Solution of fractional integro-differential equations by using fractional differential transform method. Chaos Solitons Fractals 40, 521-529 (2009)

19. Awawdeh, F., Rawashdeh, E.A., Jaradat, H.M.: Analytic solution of fractional integro-differential equations. An. Univ. Craiova, Ser. Mat. Inform. 38(1), 1-10 (2011)

20. Elbeleze, A.A., Kilıçman, A., Taib, M.T.: Approximate solution of integro-differential equation of fractional (arbitrary) order. J. King Saud Univ., Sci. 28, 61-68 (2016)

21. Elbeleze, A.A., Kilıçman, A., Taib, M.T.: Modified homotopy perturbation method for solving linear second-order Fredholm integro-differential equations. Filomat 30(7), 1823-1831 (2016)

22. Sayevand, K., Fardi, M., Moradi, E., Hemati Boroujeni, F:: Convergence analysis of homotopy perturbation method for Volterra integro-differential equations of fractional order. Alex. Eng. J. 52, 807-812 (2013)

23. Nawaz, Y: Variational iteration method and homotopy perturbation method for fourth-order fractional integro-differential equations. Comput. Math. Appl. 61, 2330-2341 (2011)

24. Yang, Y., Chen, Y., Huang, Y.: Convergence analysis of the Jacobi spectral-collocation method for fractional integro-differential equations. Acta Math. Sci. Ser. B Engl. Ed. 34(3), 673-690 (2014)

25. Ma, X., Huang, C.: Spectral collocation method for linear fractional integro-differential equations. Appl. Math. Model. 38, 1434-1448 (2014)

26. Saadatmandi, A., Dehghan, M.: A Legendre collocation method for fractional integro-differential equations. J. Vib. Control 17(13), 2050-2058 (2011)

27. Saleh, M.H., Amer, S.M., Mohamed, M.A., Abdelrhman, N.S.: Approximate solution of fractional integro-differential equation by Taylor expansion and Legendre wavelets methods. CUBO 15(3), 89-103 (2013)

28. Kumar, K., Pandey, R.K., Sharma, S.: Comparative study of three numerical schemes for fractional integro-differential equations. J. Comput. Appl. Math. 315, 287-302 (2017)

29. Nemati, S., Sedaghat, S., Mohammadi, I.: A fast numerical algorithm based on the second kind Chebyshev polynomials for fractional integro-differential equations with weakly singular kernels. J. Comput. Appl. Math. 308 231-242 (2016)

30. Maleki, M., Kajani, M.T.: Numerical approximations for Volterra's population growth model with fractional order via a multi-domain pseudospectral method. Appl. Math. Model. 39, 4300-4308 (2015)

31. Turmetov, B., Abdullaev, J.: Analytic solutions of fractional integro-differential equations of Volterra type. Int. J. Mod. Phys. Conf. Ser. 890, 012113 (2017)

32. Sahu, P.K., Saha Ray, S.: A novel Legendre wavelet Petrov-Galerkin method for fractional Volterra integro-differential equations. Comput. Math. Appl. (2016), in press. https://doi.org/10.1016/j.camwa.2016.04.042

33. Karimi Vanani, S., Aminataei, A.: Operational tau approximation for a general class of fractional integro-differential equations. Comput. Appl. Math. 30(3), 655-674 (2011)

34. Zhao, J., Xiao, J., Ford, N.J.: Collocation methods for fractional integro-differential equations with weakly singular kernels. Numer. Algorithms 65, 723-743 (2014)

35. Nazari Susahab, D., Shahmorad, S., Jahanshahi, M.: Efficient quadrature rules for solving nonlinear fractional integro-differential equations of the Hammerstein type. Appl. Math. Model. 39, 5452-5458 (2015)

36. Jiang, W., Tian, T:: Numerical solution of nonlinear Volterra integro-differential equations of fractional order by the reproducing kernel method. Appl. Math. Model. 39, 4871-4876 (2015)

37. Zhu, L., Fan, Q.: Numerical solution of nonlinear fractional-order Volterra integro-differential equations by SCW. Commun. Nonlinear Sci. Numer. Simul. 18, 1203-1213 (2013)

38. Nemati, S., Lima, P.M.: Numerical solution of nonlinear fractional integro-differential equations with weakly singular kernels via a modification of hat functions. Appl. Math. Comput. 327, 79-92 (2018)

39. Fahim, A., Fariborzi Araghi, M.A., Rashidinia, J., Jalalvand, M.: Numerical solution of Volterra partial integro-differential equations based on sinc-collocation method. Adv. Differ. Equ. 2017362 (2017)

40. Alkan, S.: A numerical method for solution of integro-differential equations of fractional order. Sakarya Üniv. Fen Bilim. Enst. Derg. 21(2), 82-89 (2017)

41. Pedas, A., Tamme, E., Vikerpuur, M.: Spline collocation for fractional weakly singular integro-differential equations. Appl. Numer. Math. 110, 204-214 (2016) 
42. Kobayashi, R., Konuma, M., Kumano, S.: FORTRAN program for a numerical solution of the nonsinglet Altarelli-Parisi equation. Comput. Phys. Commun. 86, 264-278 (1995)

43. Schoeffel, L:: An elegant and fast method to solve QCD evolution equations. Application to the determination of the gluon content of the Pomeron. Nucl. Instrum. Methods Phys. Res., Sect. A 423, 439-445 (1999)

44. Yüzbaşı, Ş.: Laguerre approach for solving pantograph-type Volterra integro-differential equations. Appl. Math. Comput. 232, 1183-1199 (2014)

45. Baykus Savasaneril, N., Sezer, M.: Laguerre polynomial solution of high-order linear Fredholm integro-differential equations. New Trends Math. Sci. 4(2), 273-284 (2016)

46. Gürbüz, B., Sezer, M., Güler, C.: Laguerre collocation method for solving Fredholm integro-differential equations with functional arguments. J. Appl. Math. 2014, Article ID 682398 (2014)

47. Al-Zubaidy, K.A.: A numerical solution of parabolic-type Volterra partial integro-differential equations by Laguerre collocation method. Int. J. Sci. Technol. 8(4), 51-55 (2013)

48. Gürbüz, B., Sezer, M.: A numerical solution of parabolic-type Volterra partial integro-differential equations by Laguerre collocation method. Int. J. Appl. Phys. Math. 7(1), 49-58 (2017)

49. Gürbüz, B., Sezer, M.: A new computational method based on Laguerre polynomials for solving certain nonlinear partial integro differential equations. Acta Phys. Pol. A 132(3), 561-563 (2017)

50. Gürbüz, B., Sezer, M.: Laguerre polynomial solutions of a class of delay partial functional differential equations. Acta Phys. Pol. A 132(3), 558-560 (2017)

51. Mahdy, A.M.S., Shwayyea, R.T.: Numerical solution of fractional integro-differential equations by least squares method and shifted Laguerre polynomials pseudo-spectral method. Int. J. Sci. Eng. Res. 7(4), 1589-1596 (2016)

52. Podlubny, I.: Fractional Differential Equations. Academic Press, San Diego (1999)

53. Herrman, R.: Fractional Calculus. World Scientific, Singapore (2014)

54. Bell, W.W.: Special Functions for Scientists and Engineers. Van Nostrand, London (1968)

55. Khader, M.M., El Danaf, T.S., Hendy, A.S.: Efficient spectral collocation method for solving multi-term fractional differential equations based on the generalized Laguerre polynomials. J. Fract. Calc. Appl. 3(13), 1-14 (2012)

56. Baleanu, D., Bhrawy, A.H., Taha, T.M.: A modified generalized Laguerre spectral method for fractional differential equations on the half line. Abstr. Appl. Anal. 2013413529 (2013)

57. Baleanu, D., Bhrawy, A.H., Taha, T.M.: Two efficient generalized Laguerre spectral algorithms for fractional initial value problems. Abstr. Appl. Anal. 2013, 546502 (2013)

58. Lebedev, N.N.: Special Functions and Their Applications. Dover, New York (1972)

\section{Submit your manuscript to a SpringerOpen ${ }^{\circ}$ journal and benefit from:}

- Convenient online submission

- Rigorous peer review

- Open access: articles freely available online

- High visibility within the field

Retaining the copyright to your article

Submit your next manuscript at $\gg$ springeropen.com 